\title{
Salivary alpha-amylase and cortisol responsiveness following electrically stimulated physical stress in bipolar disorder patients
}

This article was published in the following Dove Press journal:

Neuropsychiatric Disease and Treatment

5 December 2013

Number of times this article has been viewed

\section{Yoshihiro Tanaka \\ Yoshihiro Maruyama \\ Yoshinobu Ishitobi \\ Aimi Kawano \\ Tomoko Ando \\ Rie Ikeda \\ Ayako Inoue \\ Junko Imanaga \\ Shizuko Okamoto \\ Masayuki Kanehisa \\ Taiga Ninomiya \\ Jusen Tsuru \\ Jotaro Akiyoshi}

Department of Neuropsychiatry, Faculty of Medicine, Oita University, Hasama-Machi, Oita, Japan
Correspondence: Jotaro Akiyoshi Department of Neuropsychiatry, Faculty of Medicine, Oita University, HasamaMachi, Oita 879-5593, Japan

Tel +8I 975865823

Fax +8I 975493583

Email akiyoshi@oita-u.ac.jp
Background: Bipolar disorder (BP) is often associated with a change in hypothalamuspituitary-adrenal axis function change due to chronic stress. Salivary $\alpha$-amylase (sAA) levels increase in response to psychosocial stress and thus function as a marker of sympathoadrenal medullary system activity. However, sAA has been studied less often than salivary cortisol in BP patients.

Method: We measured Profile of Mood States and State-Trait Anxiety Inventory scores, heart rate variability, and salivary cortisol levels during electrical stimulation stress in $25 \mathrm{BP}$ patients and 22 healthy volunteers.

Results: Tension-anxiety, depression-dejection, anger-hostility, fatigue, and confusion scores in BP patients significantly increased compared with those of the healthy controls. In contrast, the vigor scores of BP patients significantly decreased compared with those of the healthy controls. Significant difference in the sAA levels was observed between BP patients and healthy controls. sAA of female patients was significantly higher than that of female healthy controls, and sAA in male patients tended to be higher than that of male healthy controls. No difference in salivary cortisol was observed between BP patients and the healthy controls. Only three time points were measured before and after the electrical stimulation stress. Furthermore, sAA secretion by BP patients increased before and after electrical stimulation.

Conclusion: These preliminary results suggest that sAA may be a useful biological marker for BP patients.

Keywords: HPA axis, bipolar disorder, $\alpha$-amylase, cortisol, SAM activity

\section{Introduction}

A number of studies ${ }^{1,2}$ have focused on the specific interaction between bipolar disorder (BP) and stress in the field of psychiatry. Extensive research has been conducted on dysregulation of the hypothalamus-pituitary-adrenal (HPA) axis and its association with $\mathrm{BP}^{3}{ }^{3}$ This dysregulation has become a promising target for drug therapy in BP patients. ${ }^{4}$ However, the role of the HPA axis in BP is poorly understood. One possibility is that the HPA axis plays an important role in dysregulated systemic cortisol metabolism.

The HPA axis plays an important role in maintaining body homeostasis in response to stress ${ }^{5,6}$ and is involved in therapy for manic episodes and depression in adults and youth. The HPA axis is also associated with an increase in cortisol levels in saliva, urine, and serum during the daytime. Overactivation of cortisol is associated with lack of suppression of adrenocorticotropic hormone release by corticotrophin-releasing hormone $(\mathrm{CRH})$ challenge, which is not inhibited by dexamethasone (DEX) challenge, and continues to occur during DEX pretreatment. ${ }^{7}$ Enhancement of the HPA axis 
activity is due to hyperactivity of $\mathrm{CRH}$ in the hypothalamus of BP patients; these findings are based on the failure of suppression with cortisol in glucocorticoid receptors (GR) in the hypothalamus and pituitary gland.

Fluoxetine, amitriptyline, desipramine, and electric shock treatment have been used to normalize CRH levels. ${ }^{8}$ However, the likelihood of relapse is high in BP patients with sustained dysregulation of the HPA axis. ${ }^{9}$ HPA hyperactivity has been used as an indicator of BP recurrence.

The sympathoadrenal medullary (SAM) is related to anxiety and awakening. ${ }^{10}$ It has been suggested that salivary $\alpha$-amylase (sAA) is an index of SAM activity, because branches of the sympathetic and the parasympathetic nerves are distributed in the salivary glands. Stimulation of sympathetic nerves increases salivary protein secretion; however, stimulation of parasympathetic nerves increases the flow of saliva. ${ }^{11}$ sAA activity is connected with the sympathetic nervous system stress response. ${ }^{12}$ Unlike most salivary analytes, such as cortisol and testosterone, that are transported from the plasma, sAA is an enzyme produced locally in the salivary glands that line the mouth. A nerve is resonated and distributed over the salivary glands by the parasympathetic nerve, and salivary secretion from various glands (situated at the bottom of the jaw and the hypoglottis at the parotid) occurs according to neurotransmitter activation. Thus, sAA is a prime candidate for specifying autonomic activity. ${ }^{13}$ Furthermore, Chatterton et $\mathrm{l}^{14}$ linked sAA to the sympathetic nervous system component of stress response. In particular, Chatterton et al suggest that the plasma norepinephrine level is related to locus coeruleus nucleus/autonomic nervous system activity and that sAA concentration can estimate the stress response in humans. Patients with borderline personality disorder have low salivary cortisol and increased sAA activity in response to psychological stress. ${ }^{15}$ Ishitobi et al recently reported the levels of sAA and cortisol in unremitted patients. ${ }^{16}$ sAA levels were positively associated with Hamilton Rating Scale for Depression ${ }^{17}$ (HAM-D) scores in unremitted patients with major depressive disorder (MDD). ${ }^{16}$ Tanaka et al also reported that sAA levels in females with MDD significantly increased compared with female controls before and after an electric stimulus, although no difference in salivary cortisol levels was observed between MDD patients and controls before or after an electric stimulus. ${ }^{18}$

In this study, we electrically activated the SAM and HPA axis in BP patients and examined the results of composite neuroendocrinology. We hypothesized a difference in sAA and cortisol responsiveness between the patients and control groups. Changes in autonomic measures were expected to parallel changes in sAA and salivary cortisol levels in BP patients.

\section{Materials and methods Participants}

Twenty-eight BP patients and 22 healthy controls participated in this study. Patients were interviewed by a psychiatrist using the semistructured interview in the Diagnostic and Statistical Manual of Mental Disorders (DSM)-IV (the Mini-International Neuropsychiatric Interview [MINI]). ${ }^{19}$ The control group comprised healthy volunteer staff members of the Oita University Hospital, Oita, Japan. Exclusion criteria for controls included the use of any medicine, present tobacco use, a history of BP, body mass index $\geq 32$, or steroid use within the last 3 years. All subjects were requested to refrain from eating within 3 hours of arrival; BP patients were also requested to refrain from taking medications within 5 hours of arrival. All subjects provided informed consent following description of the procedures and had an opportunity to ask questions about the research.

Patients with a main diagnosis of BP were enrolled. Diagnostic exclusion criteria included any acute and/or chronic medical illness as assessed by physical examination and routine laboratory testing. Patients were carefully matched with healthy control subjects for sex and age (except for the use of pharmaceutical drug treatments). A total of 22 patients were eligible; four patients did not meet the inclusion criteria. Moreover, two patients refused the procedures.

The final study sample comprised 25 patients with a main diagnosis of BP; nine of these patients had a second diagnosis of panic disorder and 12 patients suffered from social anxiety disorder. The average age of BP onset was 43.6 years (standard deviation [SD] 12.7 years). The patients were under medication at the time of testing, and these medications included selective serotonin reuptake inhibitors $(n=19)$ and selective norepinephrine reuptake inhibitors $(\mathrm{n}=3)$.

Healthy sex- and age-matched control subjects $(n=22)$ were enrolled at Oita University, Oita, Japan. Of 184 volunteers, eleven were excluded due to a present or whole-life major mental disorder according to the MINI. Of the 173 remaining volunteers, 22 were appropriate age- and sex-matched controls for the 25 patients. Table 1 shows the characteristics of the patients and their matched controls. The research protocol was approved by the ethics committee of the medical department of Oita University. 
Table I Demographic and medical characteristics by group

\begin{tabular}{llll}
\hline & Control & Bipolar & P or $\chi^{2}$ \\
\hline $\mathrm{N}$ & 22 & 25 & \\
Age (years) & $36.8 \pm 4.0$ & $43.1 \pm 13.0$ & 0.10 \\
Sex (female/male) & $11 / 1 \mathrm{I}$ & $11 / 14$ & 0.68 \\
HAM-D ${ }^{17}$ score & $1.0 \pm 1.1$ & $16.8 \pm 5.5$ & 0.00 \\
\hline
\end{tabular}

Note: Values are expressed as number, mean \pm SD.

Abbreviations: HAM-D, Hamilton Rating Scale for Depression; SD, standard deviation.

\section{Stimulation}

Subjects wore a stimulator coil on the wrist connected to a stimulator. This equipment supplied current to the motor and sensory fibers of the median nerve of the right wrist. The subject was stimulated in increasing steps until a threshold value stimulus was reached. We determined the threshold stimulus during the experiment. We gave the following instruction to the subject to determine their strongest tolerable stimulus: "If you cannot take the pain, please tell us." The strongest stimulus continued 40 seconds. The thresholds of the electric stimulus were between 16 and $27 \mathrm{~mA}$ (Table 2). Subjects were told that the level of the electric stimulus was sufficient to cause pain but would not cause any other injury. All participants were tested between 1 and $5 \mathrm{pm}$. Participants were instructed not to smoke, exercise, eat, or consume any caffeine-containing drinks at least 1 hour before testing and 1 hour after the electric stimulus was administered.

Table 2 Characteristics of bipolar disorder patients and controls

\begin{tabular}{|c|c|c|c|}
\hline & $\begin{array}{l}\text { Control } \\
(n=22)\end{array}$ & $\begin{array}{l}\text { Bipolar } \\
(n=25)\end{array}$ & $P$ \\
\hline \multicolumn{4}{|l|}{ Cormorbidity } \\
\hline Panic disorder & 0 & 9 & $<0.01$ \\
\hline Social anxiety disorder & 0 & 12 & $<0.01$ \\
\hline \multicolumn{4}{|l|}{$\mathrm{POMS}^{25}$ points } \\
\hline Tension-anxiety & $43.7 \pm 5.0$ & $65.0 \pm 13.8$ & $<0.01$ \\
\hline Depression-dejection & $45.8 \pm 5.2$ & $67.7 \pm 15.0$ & $<0.01$ \\
\hline Anger-hostility & $44.1 \pm 5.8$ & $56.0 \pm 14.5$ & $<0.0$ I \\
\hline Vigor & $54.0 \pm 13.4$ & $35.8 \pm 10.3$ & $<0.01$ \\
\hline Fatigue & $43.3 \pm 10.5$ & $62.2 \pm 13.8$ & $<0.01$ \\
\hline Confusion & $43.2 \pm 10.5$ & $66.4 \pm 18.3$ & $<0.01$ \\
\hline \multicolumn{4}{|l|}{ STA ${ }^{24}$ scores } \\
\hline Trait anxiety & $39.4 \pm 7.5$ & $56.3 \pm 14.4$ & $<0.01$ \\
\hline State anxiety & $38.0 \pm 6.5$ & $53.3 \pm 12.6$ & $<0.01$ \\
\hline Maximum electrical stimulation & $21.2 \pm 15.8$ & $23.1 \pm 18.3$ & 0.36 \\
\hline \multicolumn{4}{|l|}{ Heart rate variability } \\
\hline LF & $60.1 \pm 19.7$ & $59.3 \pm 18.4$ & 0.44 \\
\hline $\mathrm{HF}$ & $39.9 \pm 19.7$ & $40.7 \pm 18.4$ & 0.44 \\
\hline LF/HF ratio & $4.0 \pm 8.3$ & $2.5 \pm 3.0$ & 0.19 \\
\hline
\end{tabular}

Note: Data are expressed as number or mean \pm SD.

Abbreviations: HF, high-frequency; LF, low-frequency; POMS, Profile of Mood States; SD, standard deviation; STAl, State-Trait Anxiety Inventory.

\section{Statistical analysis}

We adjusted all analyses in agreement with sex and age. Data are shown as the mean \pm SD. We analyzed the data using SPSS software (version 19; IBM Corporation, Armonk, NY, USA). We used the $\chi^{2}$ test and $t$-test for the descriptive characteristics. A two-way analysis of variance followed by Dunnett's least significant difference test was used to compare sAA and cortisol responses. $P<0.05$ was considered significant.

\section{Procedures}

We measured sAA and salivary cortisol levels three times (before stimulation, immediately after stimulation and 20 minutes after stimulation), similar to previous reports. ${ }^{17,18,20}$ Exposure to the physical stress and saliva collection were performed between 1 and $5 \mathrm{pm}$ to control for circadian variations in SAA and cortisol levels. sAA was measured using the Dry Chemistry System (Nipro Corporation, Tokyo, Japan) according to the manufacturer's instructions. Saliva was sampled by directly dipping a saliva-sampling strip under the tongue for 30 seconds. ${ }^{21,22}$ The strip was immediately placed in an automatic saliva transfer system, and the saliva was transferred by compression to the $\alpha$-amylase test paper on the reverse side of the strip sleeve. The $\alpha$-amylase test paper contained the substrate 2-chloro-4-nitrophenyl4-O- $\beta$-D-galactopyranosylmaltoside (Gal- G2-CNP). The enzyme reaction was begun by transfer and compression, and free Gal-G2-CNP levels were optically measured within 20 seconds. The $\alpha$-amylase activity that reduced sugars equivalent to $1 \mu \mathrm{mol} /$ minute of maltose was defined as one unit. The concentration of salivary cortisol was analyzed by enzyme-linked immunosorbent assay, ${ }^{23}$ with intra- and interassay coefficients of variation of $3 \%$ and $10 \%$, respectively. Samples were stored at $-20^{\circ} \mathrm{C}$ until analysis.

We recorded the high- and low-frequency heart rate variability (HRV) and HRV low-/high-frequency ratio immediately after electrical stimulation by use of the APG HeartRater SA-3000P (Tokyo Iken Co, Ltd, Tokyo, Japan).

We administered the State-Trait Anxiety Inventory $(\mathrm{STAI})^{24}$ and Profile of Mood States (POMS) ${ }^{25}$ tests before conducting the electrical stimulation protocol. Furthermore, we determined the HAM-D scores before electrical stimulation. BP patients scored $\geq 8$ points on HAM-D.

\section{Results}

Tension-anxiety, depression-dejection, anger-hostility, fatigue, and confusion POMS scores in BP patients were significantly increased compared with those in healthy controls 
(Table 2). In contrast, vigor scores in BP patients were significantly decreased compared with those in healthy controls. The STAI state and trait scores significantly increased in BP patients compared with those in healthy controls. No differences were observed in any HRV measure between BP patients and healthy controls. No difference was observed in the threshold of electrical stimulation between BP patients and healthy controls (Table 2). Significant differences in sAA levels were observed between BP patients and healthy controls $\left(\mathrm{F}_{[1,45]}=6.96, P<0.01\right)$. Significantly higher sAA levels were observed in female $\mathrm{BP}$ patients versus female controls $\left(\mathrm{F}_{[1,20]}=4.38, P<0.05\right)$ (Figure 1A). A trend toward higher sAA levels was observed in male BP patients versus male controls $\left(\mathrm{F}_{[1,23]}=2.65, P=0.12\right.$ ) (Figure 1B). Finally, no differences in salivary cortisol levels were observed between BP patients and controls $\left(\mathrm{F}_{[1,45]}=0.54, P=0.48\right)$ (Figure 2).

\section{Discussion}

In the present study, we showed that an acute physical stress induced increased sAA levels in BP patients. sAA levels in BP
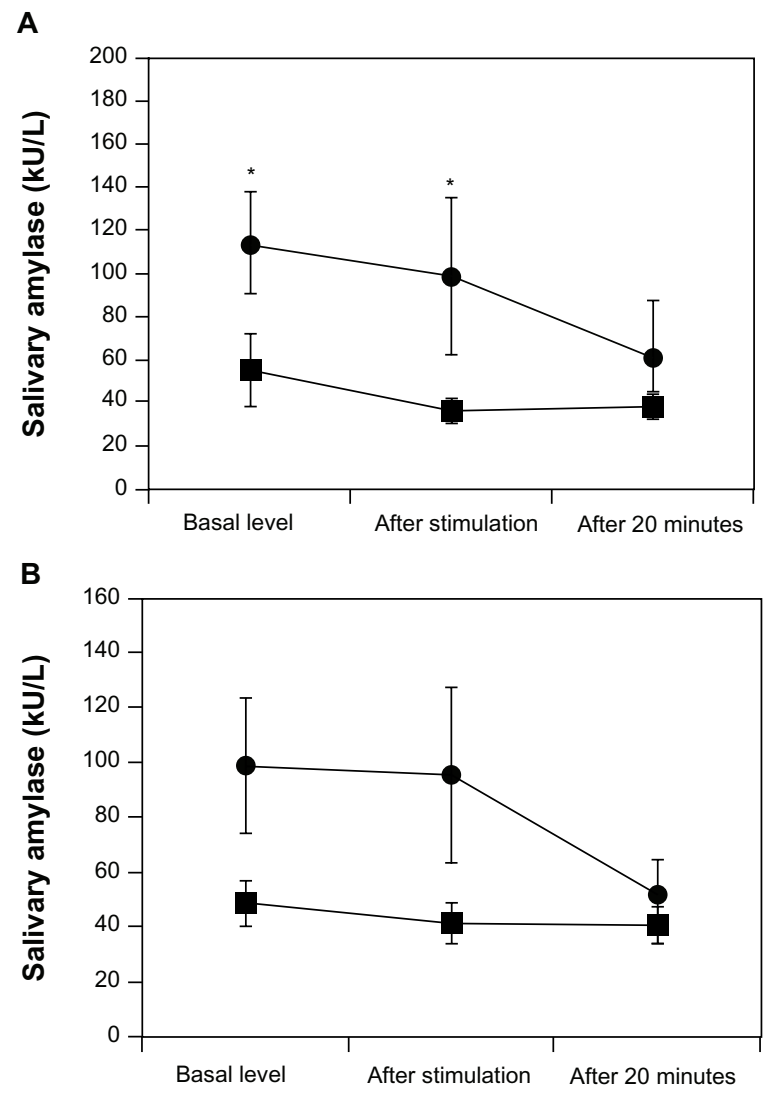

Figure I Salivary $\alpha$-amylase (sAA) responses to electrical stimulation stress in patients with bipolar disorder (BP) and healthy matched control subjects.

Notes: (A) Significantly higher sAA levels were observed in female BP patients $(\bullet)$ than in female controls ( $\mathbf{\square})$. (B) A trend toward higher sAA levels was observed in male BP patients $(\bullet)$ than in male controls $(\boldsymbol{\square})$. Values are presented as the mean \pm standard deviation. $* P<0.05$.
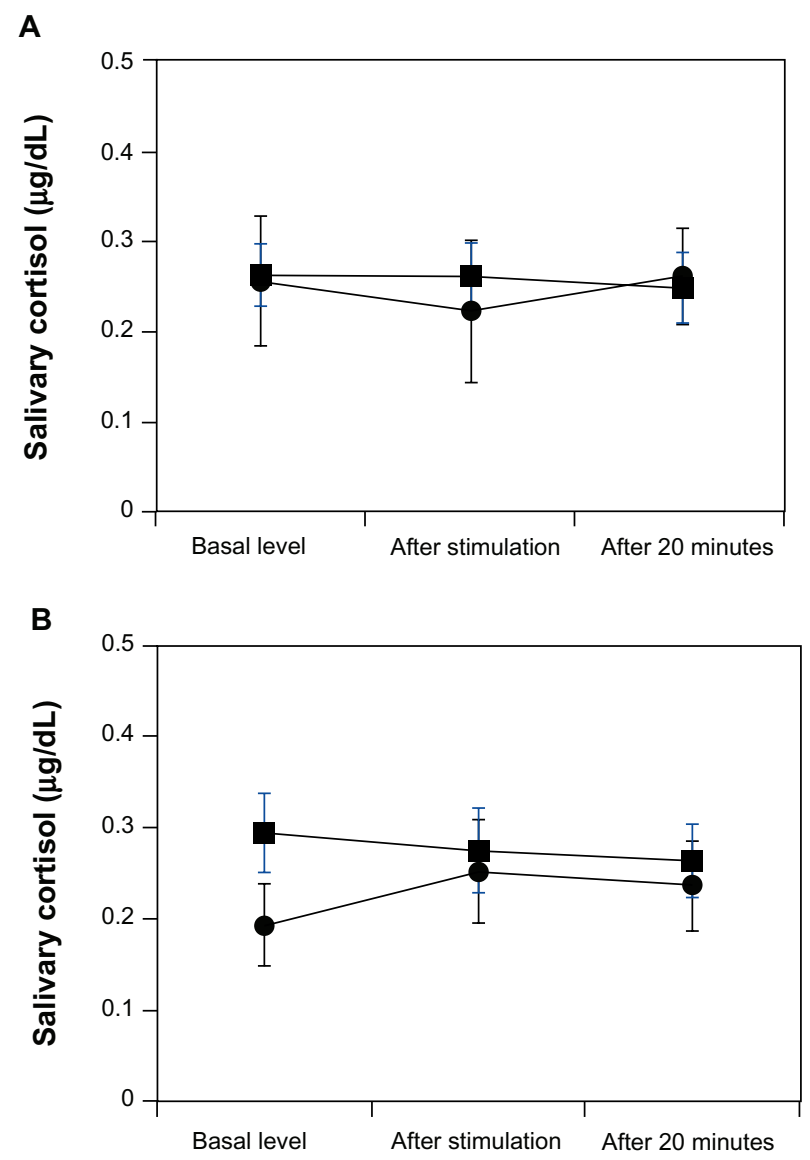

Figure 2 Salivary cortisol responses to electrical stimulation stress in patients with bipolar disorder (BP) and healthy matched control subjects.

Notes: (A) Female; (B) male. No differences in salivary cortisol levels were observed between BP patients $(\bullet)$ and controls ( $\boldsymbol{\square})$. Values are presented as the mean \pm standard deviation.

patients were significantly elevated compared with controls both before and after electrical stimulation. sAA levels have been proposed as a marker of sympathetic nervous system activity, and sAA levels also seem to be a useful measure of SAM axis activation. This study suggests a possible role of sAA before and after electrical stimulation as a potential biomarker of BP.

The influence of physical stress on sAA levels has been reported previously. ${ }^{17,18}$ In the present acute stress study, we found sAA levels were significantly higher in BP patients than in healthy controls after the stimulation. These results suggest that BP patients may experience fundamentally higher anxiety even before the electrical stimulation. Therefore, sAA levels before an electric stimulus may increase due to anticipatory anxiety in BP patients. The decrease in the vigor score in this group may also support this observation.

We considered a sex difference in the sAA response before and after the electric stimulus. sAA levels in female BP patients significantly increased compared with 
those in the female controls. A trend toward higher sAA levels before and after the stimulus was observed in male patients with BP compared with male controls. Tanaka et al previously reported that SAA levels in females with MDD are significantly higher than those in healthy controls. ${ }^{18}$ Filaire et al suggests that there are no sex differences between sAA levels and stress, ${ }^{26}$ whereas van Stegeren et al suggests that men displayed higher sAA levels compared with women during stressful tasks. ${ }^{27}$ Thus, further studies are required to examine the relationship between stress and sAA levels in BP patients of both sexes.

Differences might exist between psychological status and hormonal responses. Anticipatory anxiety presumably caused increases in sAA levels in BP patients, whereas prolonged stress suppressed SAM activity in healthy controls. In contrast, prolonged stress did not suppress SAM activity in BP patients, suggesting the maintenance of hormonal responsiveness in these individuals. However, there is not one clear reason for the observed differences between these groups. One possibility is that the duration of BP may have influenced the results. A second possibility is that individual biological or genetic backgrounds in BP patients may have affected the results, since specific genetic polymorphisms are associated with stress sensitivity. ${ }^{28,29}$ No differences in HRV values or strength of the applied electrical stimulation were observed between the two groups, so biological background did not influence the different hormonal reaction. A third possibility is that the genetic backgrounds of the depressed patients may have influenced the results. We did not check for genetic differences, such as single nucleotide polymorphisms, between the two groups; however, we will be evaluating the influence of SNPs on stress, HPA axis activity, and SAM axis activity in future studies. Further studies are necessary to evaluate the relationships between hormonal responsiveness in relation to depression and genetic composition.

We did not find any differences in salivary cortisol levels between BP patients and controls before or after electrical stimulation. HPA axis functions are usually measured by responses to the combined DEX/CRH test, the DEX suppression test, and basal cortisol levels. It has been reported that the function of the HPA axis is abnormal in BP patients. ${ }^{30}$ The DEX/CRH test clarifies dysregulated HPA axis function in both remitted and unremitted BP patients. In particular, $\mathrm{BP}$ patients show an enhanced cortisol response in the DEX/ $\mathrm{CRH}$ test in comparison with controls, and this reaction does not significantly differ between remitted and unremitted patients. ${ }^{30}$ As our participants were examined in the afternoon, between 1 and 5 pm, we may not have observed basic cortisol changes in BP patients. Furthermore, the strength of electrical stimulation may have been insufficient to change cortisol levels, as BP patients and controls in our study did not significantly differ in response to the electrical stimulation.

When we checked the differences in POMS and STAI scores between the BP and control groups, we found that the tension-anxiety, depression-dejection, anger-hostility, fatigue, and confusion scores of the POMS significantly increased in BP patients compared with those in healthy controls, whereas the vigor scores significantly decreased in BP patients. Our results agree with another study that the POMS depression-dejection scale exactly classifies persons with and without BP using the Structured Clinical Interview for DSM-IV diagnoses. ${ }^{18,30}$ STAI was chosen to evaluate state and trait anxiety. State and trait scores increased in BP patients in comparison with healthy controls, in agreement with another recent study. ${ }^{18}$ It is important to note that anticipatory anxiety might be related to increases in basal sAA levels. Finally, the Beck Depression Inventory ${ }^{31}$ and the STAI have correlation coefficients of 0.50 to $0.80,{ }^{32}$ and an ongoing depressive state may also have influenced our results.

Our study had four major limitations. One is that the number of patients and healthy controls was relatively small; further studies with a larger study sample should be performed. The second limitation was that the number of hormonal assessments was limited, so the number of tested hormonal responses should be increased in future. The third limitation is that BP patients took medication some point prior to the study, and this may have influenced the results. A fourth limitation is that we set the time period prior to the stress as the baseline, and sAA and salivary cortisol levels may have been different based on the length of time a participant had been in the hospital. ${ }^{33}$ In future studies, we will try to include patients who are not undergoing drug treatment and make comparisons between treated and nontreated patients.

\section{Conclusion}

These preliminary results suggest that sAA could be a biological marker of autonomic function in BP patients. It differentiates between sympathetic and parasympathetic responses, and has been a confounder in autonomic biomarker research. It distinguishes between the generic stress response and the possibly specific stress response associated with BP. It seemed that the current electrical stimulation paradigm did not sufficiently activate the HPA axis, and further studies should consider the strength of the applied stress. The current stress consistently activated the SAM system but was not 
strong enough to stimulate the HPA axis. Additional studies incorporating more frequent measurements and additional combinations of stress markers will be needed to examine the pathophysiology of BP patients.

\section{Acknowledgment}

The study was supported by a Grant-in-Aid for Scientific Research (C) 23591719 from the Ministry of Health and Welfare, Japan.

\section{Disclosure}

The authors report no conflicts of interest in this work. All authors declare that the funding source had no impact on the study design or the collection, analysis, and interpretation of data, writing of the report, or decision to submit the study for publication.

\section{References}

1. Steen NE, Methlie P, Lorentzen S, et al. Increased systemic cortisol metabolism in patients with schizophrenia and bipolar disorder: a mechanism for increased stress vulnerability? J Clin Psychiatry. 2011;72:1515-1521.

2. Ostiguy CS, Ellenbogen MA, Walker CD, Walker EF, Hodgins S. Sensitivity to stress among the offspring of parents with bipolar disorder: a study of daytime cortisol levels. Psychol Med. 2011;41:2447-2457.

3. Daban C, Vieta E, Mackin P, Young AH. Hypothalamic-pituitaryadrenal axis and bipolar disorder. Psychiatr Clin North Am. 2005;28:469-480.

4. Watson S, Gallagher P, Ritchie JC, Ferrier IN, Young AH. Hypothalamicpituitary-adrenal axis function in patients with bipolar disorder. $\mathrm{Br} J$ Psychiatry. 2004;184:496-502.

5. Herman JP, Ostrander MM, Mueller NK, Figueiredo H. Limbic system mechanisms of stress regulation: hypothalamo-pituitary-adrenocortical axis. Prog Neuropsychopharmacol Biol Psychiatry. 2005;29: 1201-1213.

6. Pariante CM. Glucocorticoid receptor function in vitro in patients with major depression. Stress. 2004;7:209-219.

7. Carroll BJ, Feinberg M, Greden JF, et al. A specific laboratory test for the diagnosis of melancholia. Standardization, validation, and clinical utility. Arch Gen Psychiatry. 1981;38:15-22.

8. Schüle C. Neuroendocrinological mechanisms of actions of antidepressant drugs. J Neuroendocrinol. 2007;19:213-226.

9. Zobel AW, Yassouridis A, Frieboes RM, Holsboer F. Prediction of medium-term outcome by cortisol response to the combined dexamethasone-CRH test in patients with remitted depression. Am J Psychiatry. 1999;156:949-951.

10. Aston-Jones G, Rajkowski J, Kubiak P, Alexinsky T. Locus coeruleus neurons in monkey are selectively activated by attended cues in a vigilance task. J Neurosci. 1994;14:4467-4480.

11. Baum BJ. Principles of saliva secretion. Ann N Y Acad Sci. 1993;20: 17-23.

12. Gordis EB, Granger DA, Susman EJ, Trickett PK. Salivary alpha amylase-cortisol asymmetry in maltreated youth. Horm Behav. 2008;53: 96-103.

13. Nater UM, Rohleder N, Gaab J, et al. Human salivary alpha-amylase reactivity in psychosocial stress paradigm. Int J Psychophysiol. 2005;55: $333-342$.
14. Chatterton RT Jr, Vogelsong KM, Lu YC, Ellman AB, Hudgens GA. Salivary alpha-amylase as a measure of endogenous adrenergic activity. Clin Physiol. 1996;16:433-448.

15. Nater UM, Bohus M, Abbruzzese E, et al. Increased psychological and attenuated cortisol and alpha-amylase responses to acute psychosocial stress in female patients with borderline personality disorder. Psychoneuroendocrinology. 2010;35:1565-1572.

16. Ishitobi Y, Akiyoshi J, Tanaka Y, et al. Elevated salivary $\alpha$-amylase and cortisol levels in unremitted and remitted depressed patients. Int J Psychiatry Clin Pract. 2010;14:268-273.

17. Hamilton, M. Rating depressive patients. Journal of Clinical Psychiatry. 1980;41:21-24.

18. Tanaka Y, Ishitobi Y, Maruyama Y, et al. Salivary alpha-amylase and cortisol responsiveness following electrical stimulation stress in major depressive disorder patients. Prog Neuropsychopharmacol Biol Psychiatry. 2012;36:220-224.

19. Sheehan DV, Lecrubier Y, Sheehan KH, et al. The Mini-International Neuropsychiatric Interview (MINI): the development and validation of a structured diagnostic psychiatric interview for DSM-IV and ICD-10. J Clin Psychiatry. 1998;59 Suppl 20:22-33.

20. Tanaka Y, Ishitobi Y, Maruyama Y, et al. Salivary $\alpha$-amylase and cortisol responsiveness following electrical stimulation stress in panic disorder patients. Neurosci Res. 2012;73:80-84.

21. Shetty V, Zigler C, Robles TF, Elashoff D, Yamaguchi M. Developmental validation of a point-of-care, salivary $\alpha$-amylase biosensor. Psychoneuroendocrinology. 2011;36:193-199.

22. Shetty V, Yamaguchi M. Salivary biosensors for screening traumarelated psychopathology. Oral Maxillofac Surg Clin North Am. 2010;22: 269-278.

23. Strickland PL, Deakin JF, Percival C, Dixon J, Gater RA, Goldberg DP. Bio-social origins of depression in the community. Interactions between social adversity, cortisol and serotonin neurotransmission. Br J Psychiatry. 2002;180:168-173.

24. Spielberger CD, Edwards CD, Montuori J, Lushene R. State-Trait Anxiety Inventory for Children. Menlo Park, CA: Mind Garden Inc; 1973.

25. McNair DM, Lorr M, Droppleman LF. Poms Manual: Profile of Mood States. San Diego, CA: Multi-Health Systems Inc; 1992.

26. Filaire E, Dreux B, Massart A, Nourrit B, Rama LM, Teixeira A. Salivary alpha-amylase, cortisol and chromogranin A responses to a lecture: impact of sex. Eur J Appl Physiol. 2009;106:71-77.

27. van Stegeren AH, Wolf OT, Kindt M. Salivary alpha amylase and cortisol responses to different stress tasks: impact of sex. Int $J$ Psychophysiol. 2008;69:33-40.

28. Cole SW. Elevating the perspective on human stress genomics. Psychoneuroendocrinology. 2010;35:955-962.

29. DeRijk RH, de Kloet ER, Zitman FG, van Leeuwen N. Mineralocorticoid receptor gene variants as determinants of HPA axis regulation and behavior. Endocr Dev. 2011;20:137-148.

30. Patterson K, Young C, Woods SP, Vigil O, Grant I, Atkinson JH; HIV Neurobehavioral Research Center Group. Screening for major depression in persons with HIV infection: the concurrent predictive validity of the Profile of Mood States Depression-Dejection Scale. Int J Methods Psychiatr Res. 2006;15:75-82.

31. Beck AT, Ward CH, Mendelson M, Mock J, Erbaugh J. An inventory for measuring depression. Arch Gen Psychiatry. 1961;4: 561-571.

32. Watson D, Kendall PC. Understanding anxiety and depression: Their relation to negative and positive affective states. In: Kendall PC, Watson D, editors. Anxiety and Depression: Distinctive and Overlapping Features. San Diego, CA: Academic Press; 1989:3-26.

33. Balodis IM, Wynne-Edwards KE, Olmstead MC. The other side of the curve: examining the relationship between pre-stressor physiological responses and stress reactivity. Psychoneuroendocrinology. 2010;35: 1363-1373. 
Neuropsychiatric Disease and Treatment

Dovepress

\section{Publish your work in this journal}

Neuropsychiatric Disease and Treatment is an international, peerreviewed journal of clinical therapeutics and pharmacology focusing on concise rapid reporting of clinical or pre-clinical studies on a range of neuropsychiatric and neurological disorders. This journa is indexed on PubMed Central, the 'PsycINFO' database and CAS.

The manuscript management system is completely online and includes a very quick and fair peer-review system, which is all easy to use. Visit http://www.dovepress.com/testimonials.php to read real quotes from published authors.

Submit your manuscript here: http://www.dovepress.com/neuropsychiatric-disease-and-treatment-journal 